\title{
Combined Hormonal Contraception and Women's Sexual Function: a Cross-sectional Pilot Study in a Cohort of Danish Women
}

\author{
Nanna Cassandra Læssøe ${ }^{1}$, Sarah Wåhlin ${ }^{2}$, Ellids Kristensen ${ }^{3}$, Anette Tønnes \\ Pedersen $^{4}$ and Annamaria Giraldi ${ }^{5}$ \\ 1,2,5 Department of Sexological Research, Psychiatric Center Copenhagen, Denmark \\ ${ }^{3}$ Department of Sexological Research, Psychiatric Center Copenhagen and Department of Neurology, \\ Psychiatry and Sensory Sciences, Faculty of Health Sciences, University of Copenhagen, Denmark \\ ${ }^{4}$ Department of Gynaecology and Fertilityclinic , JMC Rigshospitalet, Copenhagen University Hospital, \\ Copenhagen, Denmark.
}

Correspondence should be addressed to: Nanna Cassandra Læssøe; nacaslae@gmail.com

Received date: 20 December 2013; Accepted date: 9 April 2014 ; Published date: 1 September 2014

Academic Editor: Luciano Melo Melo Pompei

Copyright (C) 2014. Nanna Cassandra Læsse, Sarah Wåhlin, Ellids Kristensen, Anette Tønnes Pedersen and Annamaria Giraldi. Distributed under Creative Commons CC-BY 3.0

\begin{abstract}
Objective: To investigate the relationship between combined hormonal contraception (CHC) and women's sexual function with special emphasis on the type of progestin in the CHC Methods: A community sample of 252 healthy, sexually active women aged 18 to 35 completed a questionnaire including the Female Sexual Function Index (FSFI), the Female Sexual Distress Scale (FSDS), and questions about contraception use. Participants were divided into 151 users of CHC, also subdivided into progestin-type subgroups, and 101 users of non-hormonal or no contraception (non-HC). Results: Significantly fewer women using CHC reported sexual distress ( $\mathrm{p}=0.038$ ) compared to non-HC users, but the number reporting sexual problems did not differ $(\mathrm{p}=0.081)$. In subgroup analyses, significantly fewer women in the 'other progestin'-CHC group reported sexual problems $(\mathrm{p}<0.001)$ and sexual distress $(\mathrm{p}=0.008)$ compared to women in the anti-androgenic progestin-CHC group. Significantly, fewer women in the 'other progestin'-CHC group reported sexual problems $(\mathrm{p}=0.003)$ and sexual distress $(\mathrm{p}=0.006)$ versus women in the non-HC group. Results remained significant after controlling for age, relationship, children living at home, alcohol consumption, and smoking. Conclusions: Women using CHC containing anti-androgenic progestins more often reported sexual problems and sexual distress than women using CHC with other types of progestins.
\end{abstract}

Keywords: Female Sexual Dysfunction, Oral Contraception, FSFI, FSDS. 


\section{Introduction}

Hormonal contraception (HC) is prescribed in many formulations (synthetic or natural estradiol combined with different types of progestin or progestin-only preparations) and delivery systems (i.e., oral tablets, vaginal ring, transdermal patch, subcutaneous implant, and intrauterine device). Oral contraception (OC) is one of the most popular methods of contraception among young women in the western world, although with high rates of discontinuation within the first 6 months (Westhoff et al. 2007). Since the introduction of OC, reports have emerged of unwanted effects on sexual function, and negative changes in mood and sexuality predict discontinuation within the first 6 months of use (Sanders et al. 2001).

Women's sexual problems include disorders of desire, sexual arousal, lubrication, orgasm, and pain. Female sexual dysfunction is defined as sexual problems combined with sexually related personal distress (Latif and Diamond 2013). Not all women who have sexual problems report sexual distress, and sexual distress is often not measured when investigating the effects of HC on sexuality (Bancroft et al. 2003). However, it can be argued that measuring sexual distress is clinically relevant because women with sexual distress are more likely to seek professional help.

The reported negative effects of OC on sexual function have mainly been on desire and sexual thoughts, but some reports describe negative effects on lubrication and pain (Battaglia et al. 2012; Davis and Castano 2004). Research on OC effects on sexuality show varied results with reports of both improved and worsened sexual function correlated to OC. The majority of participants report no change, and the effects of OC on sexual function are therefore inconclusive (Davis \& Castano 2004).

Several bio-psychosocial factors influence women's sexual function and may contribute to the outcomes in the investigations of the effects of $\mathrm{OC}$ and other types of HC on this endpoint (Eplov et al. 2007; Lewis et al. 2010). Testosterone influences sexual function, and testosterone supplementation yields improvement in function in surgically postmenopausal women (Basaria and Dobs 2006). However, no overall relationship between low testosterone and sexual problems in women has been identified (Basson et al. 2010; Davis et al. 2005).

Combined HC (CHC) containing synthetic estradiol and progestin lowers free testosterone by suppressing ovarian androgen production and increasing sex hormone-binding globulin synthesis in the liver (Sitruk-Ware 2006). It has been hypothesized that these hormonal changes might be a possible cause of the reported negative effects of CHC on women's sexual function (Davis \& Castano 2004;Schaffir 2006). Graham et al. (2007) found some support for a relationship between the degree of reduction in the serum levels of free testosterone and the frequency of sexual thoughts; however, there was no overall evidence that the OC-induced reduction in free testosterone affected sexual function. The authors suggested that some individuals might be more sensitive to hormonal changes than others (Graham et al. 2007).

The progestins used in CHC possess partial androgenic or anti-androgenic properties and can trigger agonist as well as antagonist effects on the androgen receptor (Raudrant and Rabe 2003;Sitruk-Ware 2006). The impact on the androgen receptor could be another possible cause of the negative or positive effects of $\mathrm{CHC}$ on women's sexual function. However, the role of different progestins has received little attention even as the nature and extent of $\mathrm{HC}$ effects on women's sexual function remain unclear in despite extensive research.

Our hypothesis was that more women using CHC would have sexual problems and sexual distress than women using non-HC 
and that this relationship would be more pronounced for women using CHC formulations containing progestins with anti-androgenic properties. The aim of this study, therefore, was to compare correlations between CHC and non-HC use and sexual function, including sexual distress. We also investigated a possible correlation between CHC formulations containing progestins with anti-androgenic properties and women's sexual function and sexual distress.

\section{Methods}

\section{Participants}

The study was designed as a crosssectional investigation of healthy, sexually active women aged 18 to 35 . The participants were a subgroup from a cohort study that investigated correlations between testosterone levels and sexual function, depression and quality of life in 575 healthy women aged 18 to 65 . The study protocol was reviewed and accepted by the local Ethics Committee and the Danish Data Protection Agency. Informed consent was obtained from all participants.

The sample size needed for the study was estimated based on data from the first 100 participants of the cohort and a conservative estimate of effect. A sample size of 300 women would be enough to obtain significant differences in FSFI score between CHC and non-HC users at a confidence level of $5 \%$ and a statistical power of $95 \%$.

The participants in the cohort were recruited via advertisements posted at the University of Copenhagen, Copenhagen University Hospital, local educational centers, and local newspapers. All women in the age group 18 to 65 years who could speak and read danish were potentially eligible.

Participants in the cohort were screened by telephone. Exclusion criteria were pregnancy, nursing, or giving birth within the last 6 months; thyroid disease; pituitary disorders; polycystic ovary syndrome; diabetes; current or previous cancer; and use of antidepressants or antipsychotic drugs in the last 3 months.

Participants in the cohort attended at the Department of Gynaecology, Copenhagen University Hospital, for a short interview, measurement of weight and height, and completing the questionnaire. The questionnaire included items on basic demographic information, somatic and mental health, contraception, medicine and hormonal treatment, and the self-rating scales Female Sexual Function Index, Female Sexual Distress Scale, WHO-5 WellBeing Index, and Beck Depression Inventory II.

Participants were included in the cohort from April 2009 to November 2010. An article reporting results from the full cohort study has recently been submitted for publication.

\section{Main Outcome Measures}

\section{Female Sexual Function Index (FSFI)}

The FSFI is a 19-item, multidimensional, self-report questionnaire comprising a full scale and six domains (desire, arousal, lubrication, orgasm, satisfaction, and pain) to measure sexual function in women within the last 4 weeks (Rosen et al. 2000). Scores $\leq 26.55$ (cut-off) on the FSFI full scale represent a risk of having sexual problems (Wiegel et al. 2005). The factor structure and internal consistency have been examined and found to be satisfactory, and the FSFI has been validated to discriminate between women with sexual complaints and those without (Giraldi et al. 2011). We used the FSFI in a danish translation that had previously been back-translated (Petersen et al. 2009), and the translated FSFI questionnaire has a high degree of internal consistency (Cronbach's $\alpha=0.94$ ). Because the FSFI was developed for sexually active women, only sexually active women were included in analyses. In this study, sexual activity was measured by items 3-13 in the FSFI.

Female Sexual Distress Scale (FSDS)

The FSDS is a 12-item self-report questionnaire developed to measure 
sexually related personal distress in women. Scores $\geq 15$ (cut-off) on the FSDS indicate sexual distress, and the FSDS is a valid and reliable measure for assessing sexuality related personal distress in women (Derogatis et al. 2002). We used the FSDS in a danish translation that had previously been back-translated (Petersen et al. 2009), and the translated FSDS questionnaire has a high degree of internal consistency (Cronbach's $\alpha=0.94$ ).

\section{Manifest Female Sexual Dysfunction (MFSD)}

In this study, we used the term MFSD to describe an FSFI score below cut-off $1 \leq$ 26.55), indicating sexual problems and FSDS score above cut-off ( $\geq 15)$, indicating sexual distress, with both present at the same time (Petersen et al. 2009).

\section{Contraception and Medicine}

Combined estradiol-progestin preparations administered as oral tablets, vaginal ring, or transdermal patch were categorized as $\mathrm{CHC}$. The CHCs were further categorized based on the properties of the progestin as anti-androgenic (AAP-CHC) or other (OP-CHC). The progestins were classified according to the conventional classification (Raudrant \& Rabe 2003;Sitruk-Ware 2006). Progestin-only preparations administered as oral tablets, injections, subcutaneous implant, or hormonal IUD were categorized as progestin-only hormonal contraception (POP-HC). Condoms, diaphragm, and copper-IUD were categorized as nonhormonal contraception (non-HC).

The current intake of other medical preparations was categorized based on the current knowledge of sexual side effects as either (A) evidence of influence on women's sexual function, (B) possible influence on women's sexual function, or (C) no influence on women's sexual function (Eplov et al. 2007).

\section{Demographic Data}

The included demographic variables all correlate with women's sexual function
(Eplov et al. 2007) and were grouped as follows: age as younger (18-25 years) and older $(26-35$ years); BMI as normal $(<25)$ or overweight $(>25)$; relationship as shortterm stable relationship (0-2 years), longterm stable relationship (3 or more years), or no relationship (Klusmann 2002); 'children living at home' as some (min 1) or none (0); smoking as have smoked (currently or previously) or never smoked; alcohol consumption as low (0-13 units a week) or high (above 14 units a week), based on the recommendations of the Danish Health and Medicines Authority; and education as lower (high school or less) or higher education (more than high school).

\section{Statistical Methods}

Statistical analyses were performed using SPSS 19.0. Independent samples $t$-tests and Chi-squared tests were used for analyzing participant characteristics. Mann-Whitney test were used to compare mean FSFI and FSDS scores. Chi-squared tests were performed comparing the distribution around cut-off. Bonferroni correction was used because of multiple testing. Participants with missing data for the FSFI, FSDS, or use of contraception were excluded from the analyses.

Logistic regression analysis was performed to control significant results for confounding by factors with known influence on sexual function. Separate analyses were made with each of the following dependent variables: FSFI $\leq$ 26.55 (yes/no), FSDS $\geq 15$ (yes/no), and MFSD (yes/no). The independent variables were subgroup (OP-CHC/AAP-CHC/non$\mathrm{HC}$ ), relationship (categorized as described above), age, children living at home, alcohol consumption, and smoking.

The variables BMI, Category A and B Medicine, and Education were not included in regression analyses because these measures showed very little variation among the participants. Category C Medicine was not included because it encompassed medicine with no influence on women's sexual function (Eplov et al. 2007). 


\section{Results}

\section{Participant Characteristics}

Of the original cohort of 575 women, 313 participants were in the age group 18 to 35 and therefore eligible for this study.

Thirty participants were excluded from analyses for lack of sexual activity, missing data in the FSFI, or missing data on CHC preparations (Fig. 1). Another 11 participants were not included in the analyses because they were recent (within the last 2 months) but not current users of HC.

The remaining participants were divided into groups based on their method of contraception: 151 users of CHC, 20 users of POP-HC, and 101 users of non-HC. The CHC group was further divided into two subgroups: 27 users of AAP-CHC and 124 users of OP-CHC.
The 20 participants using POP-HC were excluded from further analyses because the aim was to compare CHC and non-HC users, and the POP-HC group could not be added to any of these groups. Thus, a total of 252 participants with a mean age of 26.3 years (SD 3.67) were included (Fig. 1). Data on socio-demographic characteristics are presented in Table 1, and data on contraception and properties of progestins in $\mathrm{CHC}$ are presented in Table 2.

The non-HC group differed from the CHC group in several characteristics: women in the non-HC group were significantly older $(p<0.001)$, were significantly less often in a stable relationship $(\mathrm{p}=0.008)$, had significantly more often children living at home $(p<0.001)$, and had significantly more alcohol consumption of more than 14 units weekly $(\mathrm{p}=0.016)$ compared with the CHC group. 


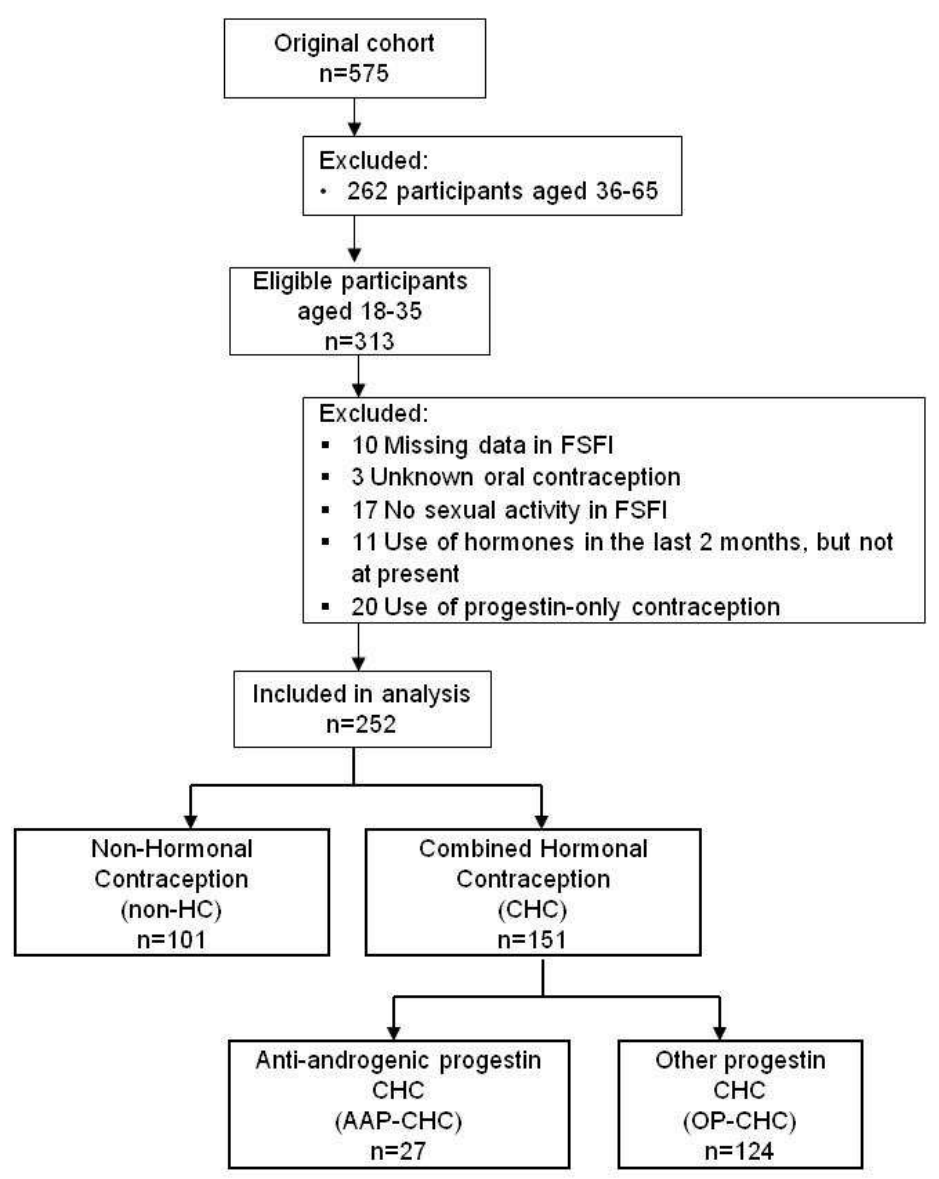

Figure 1: Flow chart of inclusion of participants and division intogroups

Table 1: Demographic data

\begin{tabular}{|l|c|c|c|}
\hline & $\begin{array}{c}\text { CHC } \\
\mathbf{n = 1 5 1}\end{array}$ & $\begin{array}{c}\text { non-HC } \\
\mathbf{n = 1 0 1}\end{array}$ & p-value \\
\hline Age mean (SD) years & $25.33(3.19)$ & $27.72(3.90)$ & $<0.001$ \\
\hline BMI mean (SD) & $22.30(2.65)$ & $22.89(2.87)$ & 0.103 \\
\hline Stable relationship & $76.8 \%$ & $61.4 \%$ & 0.008 \\
\hline Relationship duration mean (SD) years & $1.06(0.72)$ & $1.00(0.91)$ & 0.580 \\
\hline Children living at home & $2.0 \%$ & $17.0 \%$ & $<0.001$ \\
\hline Smoking currently or previous & $33.8 \%$ & $34.7 \%$ & 0.885 \\
\hline Alcohol consumption above 14 units & $4.0 \%$ & $12.0 \%$ & 0.016 \\
\hline Education high school or more & $84.8 \%$ & $87.0 \%$ & 0.621 \\
\hline Category A Medicine & $2.6 \%$ & $1.0 \%$ & 0.355 \\
\hline Category B Medicine & $1.3 \%$ & $1.0 \%$ & 0.810 \\
\hline Category C Medicine & $15.2 \%$ & $33.7 \%$ & 0.001 \\
\hline
\end{tabular}

CHC, Combined Hormonal Contraception; non-HC, non Hormonal or no Contraception; SD, standard deviation. 
Table 2: Contraception and type of progestin in the CHC

\begin{tabular}{|l|l|}
\hline \multicolumn{1}{|c|}{ Contraception } & No of participants n=252 \\
\hline No contraception & 41 \\
\hline Cobber IUD & 17 \\
\hline Diaphragm & 1 \\
\hline Condom & 42 \\
\hline Oral contraception & \\
\hline Antiandrogenic progestins & \\
\hline Cyproterone acetate & 8 \\
\hline Drospirenone & 19 \\
\hline Other Progestins & \\
\hline Levonorgestrel & 8 \\
\hline Norgestimate & 7 \\
\hline Desogestrel & 42 \\
\hline Gestodene & 43 \\
\hline Vaginal ring & \\
\hline Other Progestins & \\
\hline Etonogestrel & 23 \\
\hline Transdermal patch & \\
\hline Other Progestins & \\
\hline Norelgestromin & 1 \\
\hline
\end{tabular}

\section{Sexual Function and Sexual Distress}

Among the 252 participants, the prevalence of sexual problems (FSFI score below cut- off) was $36.1 \%$, the prevalence of sexual distress (FSDS score above cut-off) was $29.4 \%$, and the prevalence of the two combined (MFSD) was $22.2 \%$.

\section{Table 3: Scores of Female Sexual Function Index (FSFI) and Female Sexual Distress Scale (FSDS)}

\begin{tabular}{|l|l|l|l|l|l|l|}
\hline & $\begin{array}{l}\text { non-HC } \\
\mathbf{n = 1 0 1}\end{array}$ & $\begin{array}{l}\text { CHC total } \\
\mathbf{n = 1 5 1}\end{array}$ & p-value & $\begin{array}{l}\text { Subgroup } \\
\text { AAP-CHC } \\
\mathbf{n = 2 7}\end{array}$ & $\begin{array}{l}\text { Subgroup } \\
\text { OP-CHC } \\
\mathbf{n = 1 2 4}\end{array}$ & $\begin{array}{l}\mathbf{p}- \\
\text { value }\end{array}$ \\
\hline $\begin{array}{l}\text { FSFI full scale } \\
\text { mean (SD) }\end{array}$ & $\begin{array}{l}26.62 \\
(6.33)\end{array}$ & $\begin{array}{l}28.17 \\
(5.42)\end{array}$ & 0.077 & $\begin{array}{l}25,81 \\
(6,00)\end{array}$ & $\begin{array}{l}28,68 \quad(5,17) \\
\alpha\end{array}$ & 0.015 \\
\hline $\begin{array}{l}\text { FSDS mean } \\
\text { (SD) }\end{array}$ & $\begin{array}{l}9.55 \\
(10.56)\end{array}$ & 0.029 & $\begin{array}{l}13,63 \\
(10,71)\end{array}$ & $8,66(8,66) \beta$ & 0.036 \\
\hline
\end{tabular}

$\mathrm{CHC}=$ Combined Hormonal Contraception, non-HC= non Hormonal or no Contraception, AAP-CHC= $\mathrm{CHC}$ containing anti-androgenic progestin, $\mathrm{OP}-\mathrm{CHC}=\mathrm{CHC}$ containing other progestins, SD, standard deviation. $\alpha=$ non-HC vs. OP-CHC $p=0.066, \beta=$ non-HC vs. OP-CHC $p=0.019$

The FSFI full-scale scores and FSDS scores for the non-HC, CHC, and subgroups are presented in Table 3.

We found a significantly lower mean FSDS score in CHC users compared with non-HC users, but no significant differences in mean FSFI score. When stratifying the analysis by type of progestin among CHC users, we found a significantly higher FSFI score in OP-CHC users compared with AAP- 
CHC users, and a significantly lower mean FSDS score. The power at the end of the study concerning FSFI full scale was 0.522 .

\section{FSFI}

The proportions of women in the $\mathrm{CHC}$, nonHC, and subgroups with FSFI scores below cut-off indicating sexual problems are presented in Figure 2. Overall, there was no significant difference between $\mathrm{CHC}$ and non-HC users in the proportion of women who reported sexual problems. However, between the subgroups based on the type of progestin, significantly fewer women in the OP-CHC group reported sexual problems than among the non-HC users; especially, fewer women in the OP-CHC group reported sexual problems compared with women in the AAP-CHC group.

The correlations remained significant after logistic regression analyses controlling for confounding. The logistic regression analyses also showed that a relationship lasting $0-2$ years was significantly negatively correlated to FSFI score below cut-off $(\mathrm{p}<0.001)$.

\section{FSDS}

The proportions of women in the CHC, non$\mathrm{HC}$, and subgroups with FSDS scores above cut-off indicating sexual distress are presented in Figure 3. Overall, we found a significantly lower proportion of women reporting sexual distress among $\mathrm{CHC}$ users compared with non-HC users. When dividing the group into subgroups based on the type of progestin, we found a lower frequency of reported sexual distress among women in the OP-CHC group than among women in the AAP-CHC group and among non-HC users.

Logistic regression analyses did not alter these significant correlations. These analyses also showed that a stable relationship lasting 3 or more years was significantly correlated with an FSDS score above cut-off ( $\mathrm{p}=0.002)$.

\section{MFSD}

The proportions of women in the CHC, nonHC, and subgroups with MFSD indicating both sexual problems and distress are presented in Figure 4. Overall, the prevalence of manifest female sexual dysfunction did not differ between $\mathrm{CHC}$ and non-HC users; however, women in the OPCHC group had a significantly lower prevalence of MFSD compared with the non-HC users and especially compared with women in the AAP-CHC group. AAP$\mathrm{CHC}$ users and non-HC users did not differ in the frequency of MFSD.

Logistic regression analyses did not alter these significant correlations. These analyses also showed that a stable relationship lasting 3 or more years was significantly correlated with MFSD $(\mathrm{p}=0.018)$. 

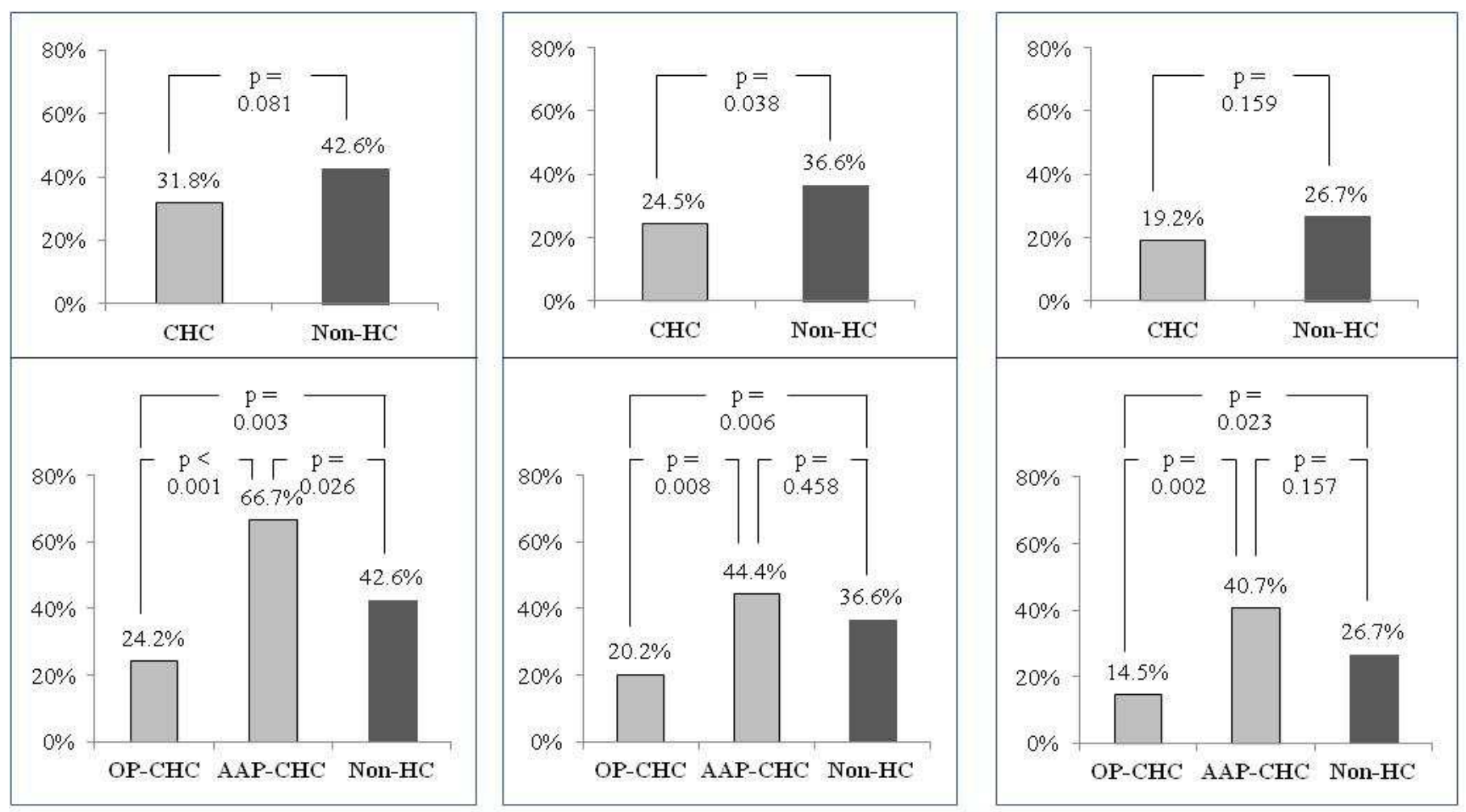

$\mathrm{CHC}$, Combined Hormonal Contraception; non-HC, non Hormonal or no Contraception; $\mathrm{OP}-\mathrm{CHC}$, Other progestin $\mathrm{CHC}$; $\mathrm{AAP}-\mathrm{CHC}, \mathrm{Anti}-\mathrm{androgenic}$ progestin $\mathrm{CHC}$.

Figure 2: Female Sexual Function Index score below cut-off ( $\leq 26.55)$
Figure 3: Female Sexual Distress Scale score above cut-off ( $\geq 15)$
Figure 4: Manifest Female Sexual Dysfunction

\section{Discussion}

The present cross-sectional study investigated the correlation between $\mathrm{CHC}$ and the frequency of sexual problems and sexual distress in sexually active women, as measured by the FSFI and FSDS questionnaires. Contrary to our hypothesis, $\mathrm{CHC}$ use was in general not correlated with a higher level of sexual problems or sexual distress in our study population. However, when stratifying the analyses according to the type of progestin, women using CHC formulations containing anti-androgenic progestins more often reported sexual problems and sexual distress than women using $\mathrm{CHC}$ with other progestins.

Only a limited number of studies have investigated the effect of hormonal contraception on sexual function in women. In an interventional study, $\mathrm{CHC}$ was associated with an increase in sexual desire measured by the FSFI Desire domain score compared to desire at baseline (Strufaldi et al. 2010). However, in a comparative interventional study, Graham et al. (2007) found no change in sexual interest compared to baseline, although there was some individual variability (Graham et al. 2007). Wallwiener et al. (2010) found lower total scale FSFI scores in women using $\mathrm{CHC}$ compared to nonusers in a cross-sectional study of German female medical students, and Higgins et al. (2008) reported an association between CHC use and less overall sexual satisfaction compared to condom users and dual ( $\mathrm{CHC}$ and condom) users in a cross-sectional study (Higgins et al. 2008; Wallwiener et al. 2010a). Thus, reports describe both positive and negative effects of $\mathrm{CHC}$ on women's sexual function.

Several methodological differences in the study design, controls, and outcomes in the mentioned studies make a comparison difficult. The limitation of the 
interventional studies is the low number of participants, whereas the larger crosssectional studies cannot estimate causation because of a lack of time dependency. Although the FSFI is widely recognized in this field of research, several other questionnaires have been used to measure women's sexual function. With the FSFI, comparison is most often based on the fullscale scores or domain scores, whereas the distribution around cut-off is not reported. FSFI domain scores may be useful when searching for a specific effect on women's sexual function, but an overall effect on sexual function can remain unnoticed if a comparison between groups is not made on the full-scale FSFI.

In this study, we investigated the different formulations of $\mathrm{CHC}$ in terms of their progestins. We found that more women using $\mathrm{CHC}$ containing anti-androgenic progestin reported sexual problems and sexual distress than women using $\mathrm{CHC}$ containing other progestins. Consistent with our results, Battaglia et al. (2012) , in an interventional study of 22 women, reported an increase in pain and a decrease in sexual function, measured using the McCoy Female Sexuality Questionnaire, after 3 months with a CHC containing an anti-androgenic progestin (Battaglia et al. 2012). In contrast, Wallwiener et al. (2010) found no difference in full-scale FSFI score between women using $\mathrm{CHC}$ containing androgenic or anti-androgenic progestins in a cross-sectional setting, and Davis et al. (2013) found equal improvement in FSFI Desire and Arousal domain scores in women randomized to CHCs containing either androgenic or anti-androgenic progestin (Davis et al. 2013; Wallwiener et al. 2010b).

Female sexual dysfunction is defined as sexual problems combined with sexually related personal distress. Among our study participants, we found an overall prevalence of possible sexual problems of $36.1 \%$ measured by FSFI, which is similar to the prevalence found in other study populations (Wallwiener et al. 2010a). We used the FSFI score cut-off because we find that it is more clinically relevant than comparing mean scores given that the cut- off identifies the participants with sexual problems. However, some women have sexual problems but are not distressed by them (Bancroft et al. 2003). To the best of our knowledge, this study is the first reported cross-sectional investigation of CHC users that has included the FSDS questionnaire to measure sexual distress. We believe that the measurement of sexually related distress is of paramount importance and clinical interest because these women are the most likely to seek help as a result of their sexual problems. Among our study participants, we found that significantly fewer CHC users reported sexual distress compared to non-HC users and that fewer women in the OP-CHC group reported sexual distress compared to both the AAP-CHC and non-HC groups. The prevalence of MFSD, which considers sexual problems and sexual distress at the same time, was lower than the prevalence of sexual problems, which is similar to the results of Shifren et al. (2008) (Shifren et al. 2008). Thus, we believe that the FSDS is a valuable measure when estimating if a change in sexual function is clinically significant.

Many factors influence women's sexual function, and we investigated some in the current work, using logistic regression analyses to control for confounding. The results showed that age, children living at home, alcohol consumption, and smoking were not correlated with women's sexual function. The correlations between women's sexual function and the groups and subgroups remained significant, but relationship status was also correlated with women's sexual function; indeed, a stable relationship is a known influential factor (Eplov et al. 2007; Klusmann 2002; Lewis et al. 2010). In our study, a short-term stable relationship (0-2 years) was significantly correlated with not having sexual problems, and a long-term stable relationship ( 3 or more years) was significantly correlated with having sexual distress. A long-term stable relationship also correlated with MFSD, the combination of sexual problems and sexual distress. One inference from these findings is that a stable relationship could have a positive effect because of greater sexual 
activity in the initial stages, but have a negative effect later in the relationship derived from worrying about partner satisfaction and other relationship problems.

Some women may be more sensitive than others to the hormonal changes induced by CHC (Graham et al. 2007). If so, it will be difficult to measure an effect on the group level, and individual measures, including hormonal levels, are needed to show whether hormonal changes are correlated with sexual function. The negative effects of CHC are possibly most important when combined with other factors that negatively influence sexual function. Longitudinal studies including blood samples could bring a better understanding of the factors that influence women's sexual function and might make it possible to estimate the actual effect of CHC.

The strengths of our study include the use of validated questionnaires and the measurement of both sexual function and sexual distress at the same time. The confounder analyses add valuable information to the importance of other factors influencing women's sexual function.

Study limitations include the fact that the cross-sectional design does not allow estimation of causation. In a cross-sectional setting, we may not reach the majority of women who experience negative effects of contraception on their sexuality because they are likely to discontinue relatively soon after starting $\mathrm{CHC}$ use. We have no information on previous contraception use or on the reason for the participants' contraceptive choice. With a mean age of 26.29 years, most women in our study may have started CHC use for the first time several years earlier, and the present users may be a selected group of women without negative experiences of $\mathrm{CHC}$ use. The participants in this cohort had in general a

\section{References}

1. Bancroft, J., Loftus, J., \& Long, J.S. 2003. Distress about sex: a national survey of women in heterosexual relationships. Arch.Sex Behav., 32, (3) 193-208 higher degree of education than the background population, which could affect the external validity of the results. The stratification into subgroups was based on the hormonal formulations and not on the administration of the contraception, although it is possible that the administration may also influence women's sexual function. Regarding the AAP-CHC group, a limitation is that some CHCs containing anti-androgenic progestin are used in treating acne and hirsutism, which may also influence women's sexual function. However, women with polycystic ovary syndrome were not included in the cohort. Also, the AAP-CHC group was considerably smaller than the other groups.

Although this study has limitations, we believe it is valuable because few other studies have investigated the relationship between women's sexual function and different CHC formulations. The present results add to the existing knowledge on the sexual function and the use of contraceptives.

In conclusion, the findings of this crosssectional study of sexually active women suggest that $\mathrm{CHC}$ use in general is not correlated with a higher degree of sexual problems or sexual distress. However, women in this study population using CHC formulations containing anti-androgenic progestins more often reported sexual problems and sexual distress measured by FSFI and FSDS than women using CHC with other types of progestins. Further research and longitudinally controlled studies, including blood samples, are needed to reveal the nature and extent of $\mathrm{CHC}$ effects on women's sexual function. CHC is still one of the most used types of contraception, and our results do not suggest that this fact should be a cause of concern. However, if a woman using CHC complains of sexual problems, we would suggest a change in CHC formulation to a CHC without an antiandrogenic progestin or to a non-HC.

2. Basaria, S. \& Dobs, A.S. 2006. Clinical review: Controversies regarding transdermal androgen therapy in postmenopausal women. $J$ Clin Endocrinol.Metab, 91, (12) 4743-4752 
3. Basson, R., Brotto, L.A., Petkau, A.J., \& Labrie, F. 2010. Role of androgens in women's sexual dysfunction. Menopause., 17, (5) 962-971

4. Battaglia, C., Battaglia, B., Mancini, F., Busacchi, P., Paganotto, M.C., Morotti, E., \& Venturoli, S. 2012. Sexual behavior and oral contraception: a pilot study. J.Sex Med., 9, (2) 550-557

5. Davis, A.R. \& Castano, P.M. 2004. Oral contraceptives and libido in women. Annu.Rev Sex Res, 15, 297-320

6. Davis, S.R., Bitzer, J., Giraldi, A., Palacios, S., Parke, S., Serrani, M., Mellinger, U., \& Nappi, R.E. 2013. Change to Either a Nonandrogenic or Androgenic ProgestinContaining Oral Contraceptive Preparation is Associated with Improved Sexual Function in Women with Oral Contraceptive-Associated Sexual Dysfunction. J.Sex Med., 10, (12), 30693079

7. Davis, S.R., Davison, S.L., Donath, S., \& Bell, R.J. 2005. Circulating androgen levels and self-reported sexual function in women. JAMA, 294, (1) 91-96

8. Derogatis, L.R., Rosen, R., Leiblum, S., Burnett, A., \& Heiman, J. 2002. The Female Sexual Distress Scale (FSDS): initial validation of a standardized scale for assessment of sexually related personal distress in women. J.Sex Marital Ther., 28, (4) 317-330

9. Eplov, L., Giraldi, A., Davidsen, M., Garde, K., \& Kamper-Jorgensen, F. 2007. Sexual desire in a nationally representative Danish population. J.Sex Med., 4, (1) 47-56

10. Giraldi, A., Rellini, A., Pfaus, J.G., Bitzer, J., Laan, E., Jannini, E.A., \& FuglMeyer, A.R. 2011. Questionnaires for assessment of female sexual dysfunction: a review and proposal for a standardized screener. J.Sex Med., 8, (10) 2681-2706

11. Graham, C.A., Bancroft, J., Doll, H.A., Greco, T., \& Tanner, A. 2007. Does oral contraceptive-induced reduction in free testosterone adversely affect the sexuality or mood of women?

Psychoneuroendocrinology, 32, (3) 246-255

12. Higgins, J.A., Hoffman, S., Graham, C.A., \& Sanders, S.A. 2008. Relationships between condoms, hormonal methods, and sexual pleasure and satisfaction: an exploratory analysis from the Women's Well-Being and Sexuality Study. Sex Health, 5, (4) 321-330

13. Klusmann, D. 2002. Sexual motivation and the duration of partnership. Arch.Sex Behav., 31, (3) 275-287

14. Latif, E.Z. \& Diamond, M.P. 2013. Arriving at the diagnosis of female sexual dysfunction. Fertil.Steril., 100, (4), 898-904

15. Lewis, R.W., Fugl-Meyer, K.S., Corona, G., Hayes, R.D., Laumann, E.O., Moreira, E.D., Jr., Rellini, A.H., \& Segraves, T. 2010. Definitions/epidemiology/risk factors for sexual dysfunction. J.Sex Med., 7, (4 Pt 2) 1598-1607

16. Petersen, C.D., Giraldi, A., Lundvall, L., \& Kristensen, E. 2009. Botulinum toxin type A-a novel treatment for provoked vestibulodynia? Results from a randomized, placebo controlled, double blinded study. J.Sex Med., 6, (9) 2523-2537

17. Raudrant, D. \& Rabe, T. 2003. Progestogens with antiandrogenic properties. Drugs, 63, (5) 463-492

18. Rosen, R., Brown, C., Heiman, J., Leiblum, S., Meston, C., Shabsigh, R., Ferguson, D., \& D'Agostino, R., Jr. 2000. The Female Sexual Function Index (FSFI): a multidimensional self-report instrument for the assessment of female sexual function. J.Sex Marital Ther., 26, (2) 191208

19. Sanders, S.A., Graham, C.A., Bass, J.L., \& Bancroft, J. 2001. A prospective study of the effects of oral contraceptives on sexuality and well-being and their relationship to discontinuation. Contraception, 64, (1) 51-58 
20. Schaffir, J. 2006. Hormonal contraception and sexual desire: a critical review. J Sex Marital Ther., 32, (4) 305-314

21. Shifren, J.L., Monz, B.U., Russo, P.A., Segreti, A., \& Johannes, C.B. 2008. Sexual problems and distress in United States women: prevalence and correlates. Obstet.Gynecol., 112, (5) 970-978

22. Sitruk-Ware, R. 2006. New progestagens for contraceptive use. Hum.Reprod.Update., 12, (2) 169-178

23. Strufaldi, R., Pompei, L.M., Steiner, M.L., Cunha, E.P., Ferreira, J.A., Peixoto, S., \& Fernandes, C.E. 2010. Effects of two combined hormonal contraceptives with the same composition and different doses on female sexual function and plasma androgen levels. Contraception, 82, (2) 147154

24. Wallwiener, C.W., Wallwiener, L.M., Seeger, H., Muck, A.O., Bitzer, J., \& Wallwiener, M. 2010a. Prevalence of Sexual
Dysfunction and Impact of Contraception in Female German Medical Students. J.Sex Med., 7, (6), 2139-2148

25. Wallwiener, M., Wallwiener, L.M., Seeger, H., Mueck, A.O., Zipfel, S., Bitzer, J., \& Wallwiener, C.W. 2010b. Effects of sex hormones in oral contraceptives on the female sexual function score: a study in German female medical students. Contraception, 82, (2) 155-159

26. Westhoff, C.L., Heartwell, S., Edwards, S., Zieman, M., Stuart, G., Cwiak, C., Davis, A., Robilotto, T., Cushman, L., \& Kalmuss, D. 2007. Oral contraceptive discontinuation: do side effects matter? Am J Obstet.Gynecol., 196, (4) 412-416

27. Wiegel, M., Meston, C., \& Rosen, R. 2005. The female sexual function index (FSFI): cross-validation and development of clinical cutoff scores. J.Sex Marital Ther., $31,(1) 1-20$ 